\title{
Is the frequency of adult strabismus surgery increasing?
}

\author{
Andrew T Astle*1, Tom Foulsham², Alexander J Foss ${ }^{3} \&$ Paul V. McGraw ${ }^{1}$ \\ 1. Visual Neuroscience Group, School of Psychology, University of Nottingham, Nottingham, UK \\ 2. Department of Psychology, University of Essex, Colchester, Essex, UK \\ 3. Department of Ophthalmology, University Hospital, Queen's Medical Centre, Nottingham, UK \\ *corresponding author contact details: \\ Dr Andrew T Astle \\ School of Psychology \\ University of Nottingham \\ University Park \\ Nottingham \\ NG7 2RD \\ United Kingdom \\ andrew.astle@nottingham.ac.uk
}

Tel: 01158467386

Running head: The frequency of adult strabismus surgery

Keywords: strabismus, heterotropia, surgery, amblyopia

Disclosures: The authors report no conflicts of interest and have no proprietary interest in any of the materials mentioned in this article.

Acknowledgements: ATA is funded by a National Institute for Health Research (NIHR) Postdoctoral Fellowship. This report presents independent research funded by the NIHR. The views expressed are those of the authors and not necessarily those of the NHS, the NIHR or the Department of Health. The authors thank Professor Alistair Fielder for useful comments on an earlier version of the manuscript.

Contributorship statement: Conceptualisation, A.T.A., T.F., A.J.F., and P.V.M.; Methodology, A.T.A. and P.V.M.; Formal Analysis, A.T.A.; Visualisation, A.T.A.; Writing - Original Draft, A.T.A.; Writing - Review \& Editing, A.T.A., T.F., A.J.F., and P.V.M.; Funding Acquisition, A.T.A.

Words: 2924, Tables: 0, Figures: 4

Accepted for publication in Ophthalmic \& Physiological Optics 


\section{Abstract}

\section{Purpose}

In recent years there has been an increase in evidence for the functional and psychosocial benefits of correcting strabismus/heterotropia in adults. This study aimed to establish whether there has been an associated change in the frequency of strabismus surgery performed on adults in England since 2000.

\section{Methods}

Data on strabismus surgery performed in England between 2000 and 2014 were obtained from Hospital Episode Statistics, Health and Social Care Information Centre, England. The frequency of strabismus surgery was analysed for different age groups. Data were considered in the context of total population data for England, obtained from the Office for National Statistics.

\section{Results}

There was little change in the total number of strabismus operations performed in 2000 to 2014 (1\% reduction). In the same period the number of operations performed on children aged 0-15 years decreased by $17 \%$. In contrast, there was a $24 \%$ increase in the number of strabismus operations performed on patients aged 15 years or older.

\section{Conclusions}

Although strabismus surgery is still most commonly performed on children, the data show there has been a significant increase in the number of strabismus operations performed on adults. We speculate that this increase is connected to the growing weight of evidence detailing the functional and psychosocial consequences of strabismus. These results have potential implications for the delivery of future care. 


\section{Introduction}

Strabismus, an abnormal alignment of the eyes, affects approximately $4 \%{ }^{1}$ of people and results in a number of negative outcomes. Depending on the age of onset, functional deficits can include diplopia and visual confusion, asthenopia, an abnormal head posture, reduced depth perception, and a reduction in the size of the visual field in cases of esotropia. ${ }^{2}$ In addition, strabismus has significant psychosocial costs. For example, communication, interpersonal relationships, employment prospects, finding a partner, self-image and mental health are detrimentally affected by strabismus. ${ }^{3}$ Strabismus also reduces quality of life and the majority of patients with this condition would be willing to trade part of their life expectancy to have it corrected. ${ }^{4}$

Current evidence suggests that adult strabismus surgery is safe, effective $\mathrm{e}^{2}$ and can lead to functional improvements. As well as eliminating diplopia, correction of adult strabismus can lead to improvements in visual function such as binocular summation, ${ }^{5}$ fusion, ${ }^{6}$ and stereopsis. ${ }^{7}$ Importantly, surgery in adults has also been shown to reduce the psychosocial consequences of strabismus. ${ }^{3}$

Given the growing evidence documenting both the negative consequences of strabismus, and benefits of strabismus surgery in adults to rectify it, we wondered whether there has been an accompanying rise in the frequency of strabismus surgery in adults. Several studies have found a decline in the frequency of paediatric strabismus surgery. ${ }^{8-12}$ Other studies have looked at the prevalence of strabismus surgery in patients over $60^{13,14}$ or 65 years of age. ${ }^{15,16}$ To our knowledge, however, there are no studies that have investigated recent trends in the frequency of strabismus surgery undertaken in adults as a whole. In this retrospective study, we determined whether there was a change in the frequency of strabismus surgery performed on adults in England, comparing rates to those for children, between 2000 and 2014 by analysing Hospital Episode Statistics.

\section{Materials and methods}

We obtained data on strabismus surgery performed on admitted patients from 2000 to 2014 from Hospital Episode Statistics (HES), Health and Social Care Information Centre (HSCIC), England. ${ }^{17}$ These statistics consist of data from National Health Service (NHS) hospitals in England. They include data for private patients treated in NHS hospitals and patients who had care delivered by treatment centres funded by the NHS. However, the statistics do not include data for patients treated privately in private hospitals.

We analysed the number of finished consultant episodes (FCE's) relating to strabismus surgery on patients less than 15 years of age and on patients aged 15 years and older. FCE's consist of a count on the number of HES records related to admitted patient care that ended during a given year. These records can each have up to 24 interventions or procedures recorded. We included data from primary/main procedures only, which reflect the most resource intensive procedure performed during the episode. Data for a given year comprise episodes for the 12-month period ending in March of that year. 
Procedures are recorded using the Office of Population Censuses and Surveys Classification of Surgical Operations and Procedures (4th revision) classification system (OPCS-4). This is an NHS Fundamental Information Standard, which allows reporting of information at different levels of granularity. Procedure groups can be identified by 3 character codes or more specific 4 character codes.

First, we analysed the number of strabismus operations performed, regardless of the type of strabismus. There were six 3-character codes that corresponded to strabismus surgery: C31 Combined operations on muscles of eye, C32 Recession of muscle of eye, C33 Resection on muscle of eye, C34 Partial division of tendon of muscle of eye, C35 Other adjustment to muscle of eye, C37 Other operations on muscle of eye. We analysed the number of FCE's relating to these six codes. In a separate analysis, data on the number FCE's relating to surgery on the lateral and medial rectus muscles was analysed using 4-character codes.

Changes in the total number of procedures performed may be due to changes in the size of the population. Therefore, data on the number of strabismus operations performed were also considered in the context of the total population of the appropriate group. Population data for England for each age group were obtained from Annual Mid-year Population Estimates for years 2000-2014 from the Office for National Statistics. ${ }^{18}$ This takes into account births, deaths, immigration and emigration to estimate the number of people living in England, regardless of nationality.

\section{Results}

\section{Number of strabismus operations}

Our analysis included a total of 177,616 finished consultant episodes for strabismus surgery. The total number of strabismus operations performed changed from 12,351 in 2000 to 12,199 in 2014, a difference of only 1\% (panel A, Figure 1). In the same period, for patients aged younger than 15 years of age, the number of operations reduced by $17 \%$, from 7554 to 6269 , and for patients aged 15 years and older, increased by 24\%, from 4797 to 5930 (panel B, Figure 1). The relative proportion of strabismus surgery performed on children:adults changed from approximately 3:2 in 2000 to 1:1 in 2014. 

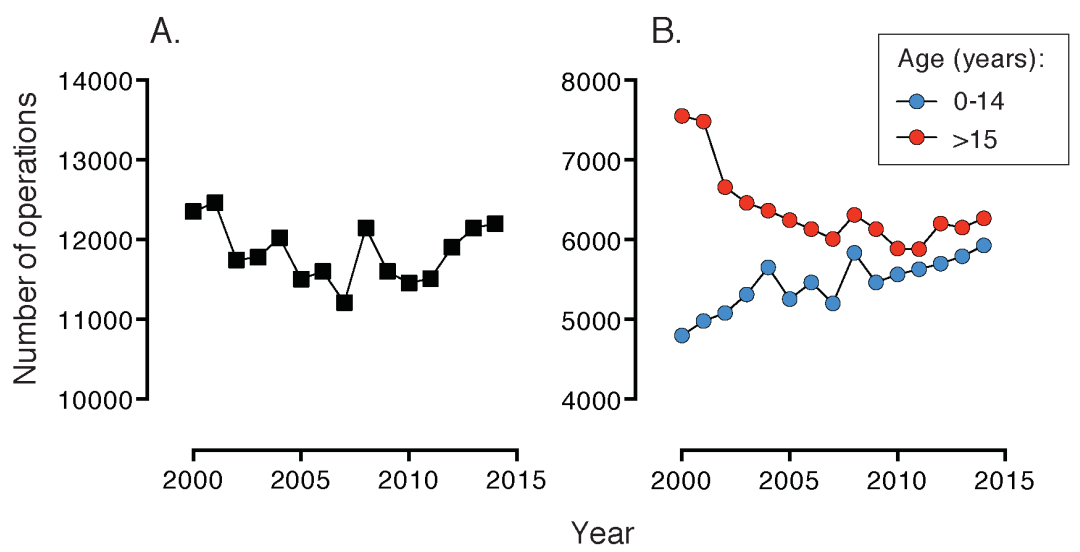

Figure 1. Strabismus operations 2000 - 2014. A) Total number of strabismus operations B) Strabismus operations on patients aged 0-14 years and patients aged 15 years and older.

\section{Population change in England}

We wondered whether the change in the number of strabismus operations undertaken could be explained by changes in population size. From 2000 to 2014 , there was an increase of $10 \%$ (49.23 to 54.32 million) of the total population, an increase of $3 \%$ of people aged $0-14$ years ( 9.36 to 9.68 million), and increase of $12 \%$ of people aged 15 years or older (39.88 to 44.64 million). See Figure 2. The change in the number of strabismus operations cannot be fully explained by the change in the relevant group sizes, since the change in population size was not equivalent to the change in number of operations for any group (e.g. 24\% increase in number of strabismus operations in patients aged 15 years and older, but only $12 \%$ increase in the total population for that group).

A.

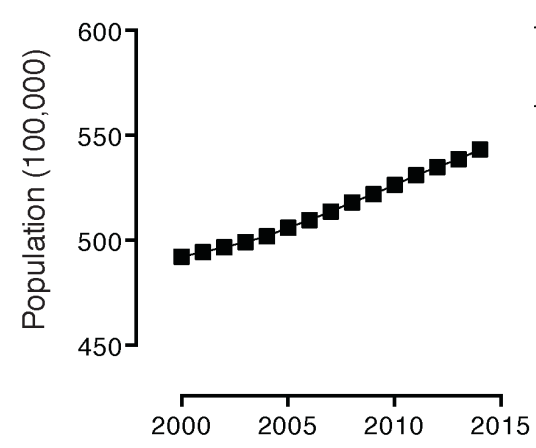

B.

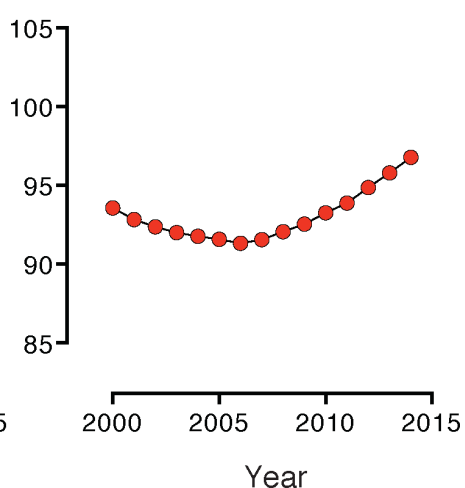

C.

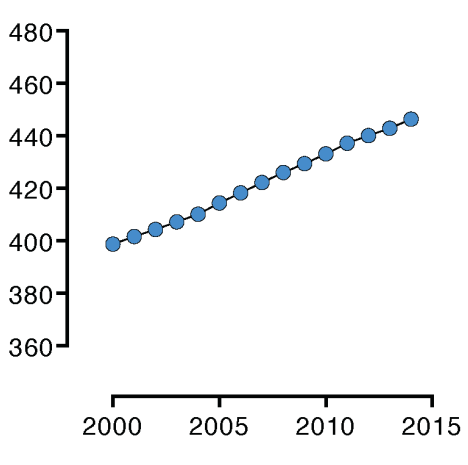

Figure 2. Estimated population of England from 2000 to 2014 for A) all ages B) patients aged under 15 years and C) patients aged 15 years or older. Data from the Annual Mid-Year Population Estimates, Office for National Statistics. 
To better visualise the data with respect to population, the number of strabismus operations per 100,000 population was calculated for each year between 2000 and 2014 (Figure 3). The number of strabismus operations per 100,000 population decreased by $10 \%$ (from 25.09 to 22.46 million) when considering patients of all ages and decreased by $20 \%$ (from 80.74 to 64.79 million) for patients younger than 15 years. However, for patients aged 15 years and older, there was an increase of $10 \%$ (12.03 from to 13.28).

A.

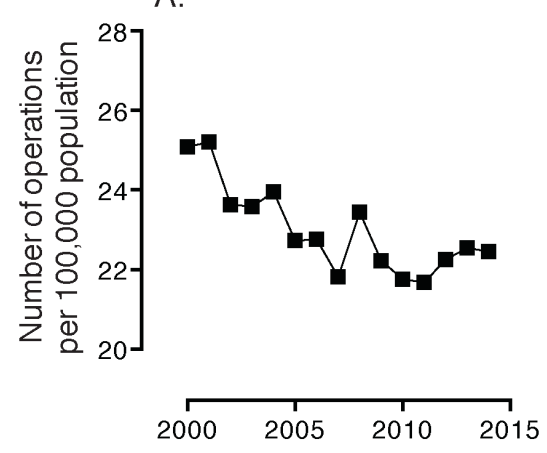

B.

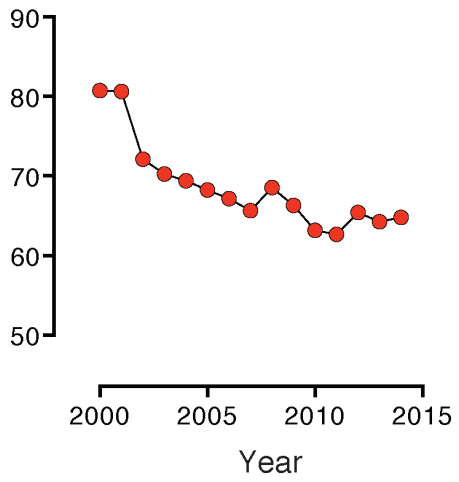

C

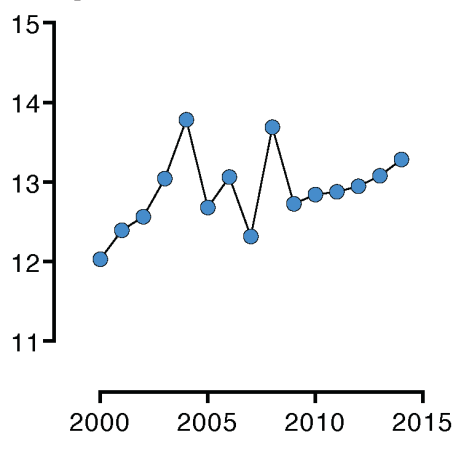

Figure 3. The number of strabismus operations per 100,000 population of England 2000 to 2014. Panel A shows data for all age groups. Panels B and C show data for patients aged 0-14 years and patients aged 15 years and older respectively.

\section{Operations for horizontal deviations}

$90 \%$ of adults who undergo surgery for strabismus that develops before visual maturity have a horizontal deviation, compared to $66 \%$ of patients who develop strabismus after visual maturity. ${ }^{19}$ Reconstructive surgery to correct for longstanding strabismus in adults, therefore, will comprise of operations for horizontal deviations in the vast majority of cases. Therefore, in a separate analysis, we considered operations on the medial and lateral rectus muscles, which are primarily responsible for horizontal eye movements. We analysed FCE's for 4-character codes related to surgery on these muscles. No data on FCE's for code 31.6 were available for years 2007 or earlier. Therefore, we restricted our analysis to the years 2008-2014. The number of operations on the medial and lateral recti per 100,000 population increased by $10 \%$ (7.20 to 7.93 ) in patients 15 years or older during this period. In contrast, there was little change for patients aged 0-14 years (53.22 to 53.75 ).

\section{Change in the number of strabismus diagnoses}

HES data contains information on diagnosis. There are two 3-character International Classification of Diseases (ICD) codes related to diagnosis of strabismus: H49 Paralytic strabismus and H50 Other strabismus. From 2000 to 2014 , for patients aged 15 years or older, the number of primary diagnoses (the primary reason why a patient was treated) increased for both of these ICD codes: from 798 to 1731 for paralytic strabismus and from 4364 to 5840 for other strabismus. From the separate HES Diagnosis and HES Procedures \& Interventions data it is not possible to determine which of these patients went on to have a given procedure. Therefore, it's not possible to say with any certainty whether the increase in the number of strabismus surgeries undertaken on 
patients over 15 years old was due to an increase in a particular diagnosis of strabismus.

\section{Distribution of ages at which strabismus surgery is performed}

HES data are divided into different age bands and it is not possible to split data into groups that encompass all children (younger than 16 years) and all adults (older than 16 years). Instead, data are separated into age 0-14 years and patients aged 15 years or older. This issue is relevant to studies that have investigated changes in the frequency of paediatric strabismus surgery because they don't include figures for all children ${ }^{9,10,12}$. It also means that it's theoretically possible that the increases we observe for strabismus surgery beyond 15 years old could be driven by changes in surgery rates for children aged 15 years. However, we are confident this is not the case for the following reasons.

First, we further analysed data from patients aged 15 years and older, dividing the data into subgroups: patients aged 15-59, 60-74 and 75 years and over. There was an increase in the number of strabismus operations undertaken in each of these groups from 2000 to 2014 , even when changes in the total population of each group were taken into account. In fact, the largest (23\%) increase was for the 60-74 age group.

Second, for 2013 and 2014, we were able to further sub-divide the data so that the number of strabismus operations undertaken on patients aged $15,16,17,18$, and 19 years of age could be viewed in isolation. These data are shown in Figure 4. For all other patients, data are shown in 5-year age bands. The numbers of operations performed on patients aged 15 years of age are a small fraction of the total number of strabismus operations performed on adults (1\% in 2014). We therefore believe that the increase in the number of operations performed on patients aged 15 years and older reflects the true underlying pattern of data for adult surgeries as a whole. 


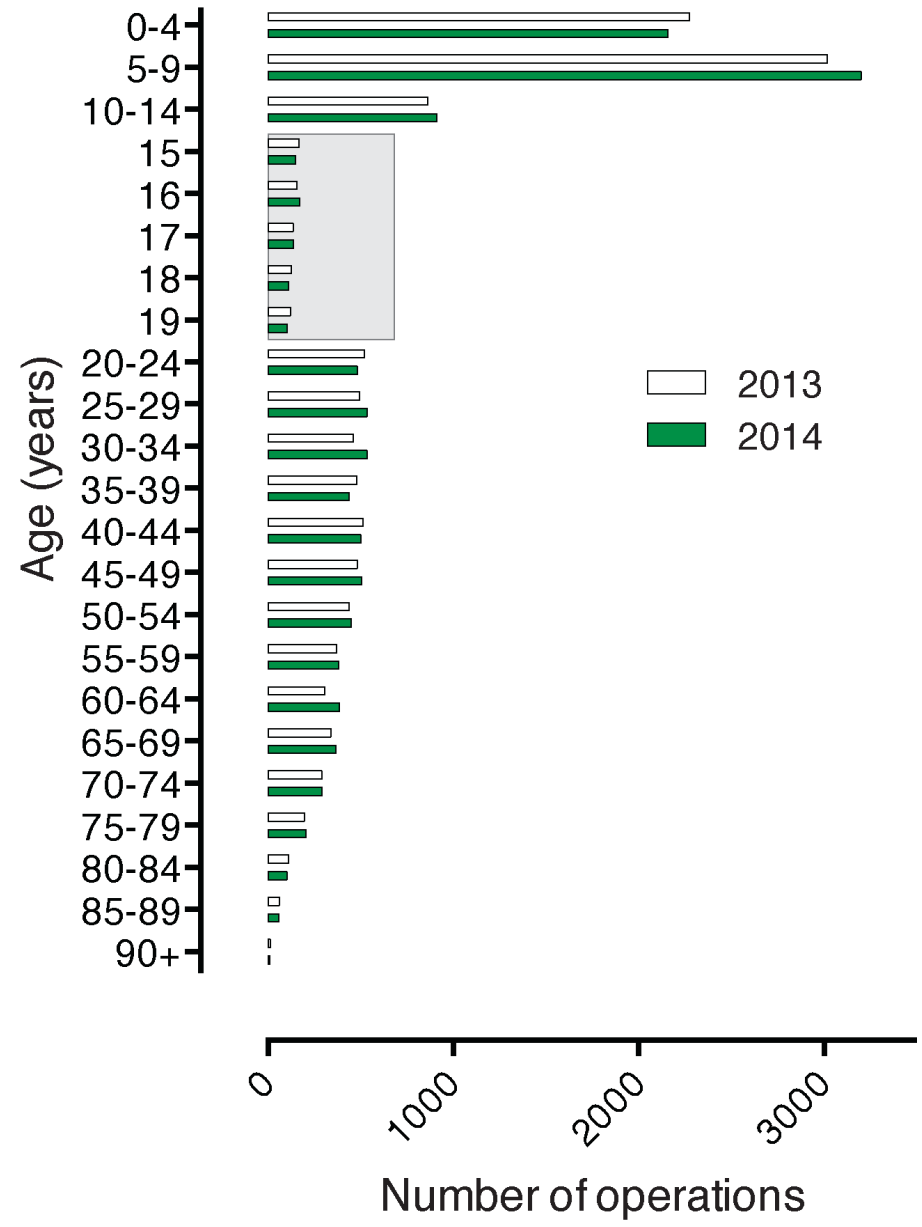

Figure 4. The number of strabismus operations performed on patients in 2013 (white bars) and 2014 (green bars). Data are divided into 5-year age bands apart from data for patients aged 15 to 19 years of age, which are divided into single year bands. The length of the grey bar denotes the number of operations undertaken in all patients aged 15-19 years in 2014.

\section{Discussion}

Our results provide important information on the change in rates of strabismus surgery performed on adults in England. A decrease in paediatric strabismus surgery was roughly balanced by an increase in the number of strabismus operations performed on adults, leading to little change in the overall number of strabismus operations performed between 2000 and 2014.

The reduction in the amount of strabismus surgery performed on children over the 14-year period examined is consistent with previous studies, which also found a decline in paediatric surgery in earlier time periods in England, 8, 9, 11, 12 Scotland, ${ }^{9,10}$ and Canada ${ }^{11}$ (although this doesn't appear to be the case in the United States ${ }^{20}$ ). Paediatric strabismus surgery appears to have been declining from at least as early as 1968: One study that analysed the annual admissions rates for strabismus surgery performed on children aged 0-15 in England found a decrease from 189 episodes per 100,000 population in 1968 to 64 episodes per 100,000 population in $2010 .^{12}$ 
Is the rate of decline in paediatric strabismus surgery changing? Arora et al., found that strabismus surgery performed on children aged 0-16 declined by 41\% in England between 1989 and 2000 from 15,083 to 8,869.11 This equates to a reduction of approximately 565 operations per year. We found a reduction of 92 operations per year from 2000 to 2014, suggesting a slowing in the rate of decline of paediatric strabismus surgery.

If the rate of decline in paediatric strabismus surgery is indeed slowing, the situation may arise where the total number of strabismus operations performed increases due to a continued increase in adult strabismus surgery. This situation may be likely, given that the population increase could only explain approximately half the increase in the number of adult strabismus operations.

A study investigating the reasons for delay of strabismus surgery in adults found that patients with strabismus developing before visual maturation delay surgery by 29 years, while those patients with strabismus developing after visual maturation delay surgery by 10 years on average. ${ }^{21}$ The reasons for delaying treatment included surgery not being offered, patients being told that nothing could be done to correct their strabismus or that they were too old for surgery. In addition, some patients reported that they, or their parents, declined surgery when it was offered. Delay of treatment from childhood to adulthood would explain both the decrease in strabismus surgery for children and the increase in adult strabismus surgery that we found. Parents may decline surgery for their children so that they can make an informed decision for themselves as an adult. Carrying out strabismus surgery in adulthood has the advantage that cooperation is greater and responses to behavioural measurements more reliable, leading to better accuracy. Although the risk of developing diplopia following surgery does not appear to depend on the duration of strabismus, ${ }^{22}$ delaying treatment carries an increased risk of amblyopia development and reduced binocularity. ${ }^{23}$ In addition, delaying treatment extends the duration of time during which patients are likely to suffer from the negative psychosocial effects of the ocular misalignment.

The increase in the frequency of adult strabismus surgery may be driven by an erosion of some of the barriers that delay strabismus surgery in adults. ${ }^{21}$ For example, better education leading to eye care professionals and patients being more aware of the low risks and potential benefits of surgery, and an increasing willingness to consider surgery, may have contributed to the rise.

Another potential reason for the rise in frequency of adult strabismus surgery is a change in expectations. The main expectation of adults due to undergo strabismus surgery is an improvement in appearance 24,25 - something partly determined by societal values - followed by correction of diplopia and improvements in vision. ${ }^{25}$ Over the last decade, there has been an increase in the number of operations performed in the UK to improve appearance. ${ }^{26}$ We suggest that an increase in the number of adults actively seeking reconstructive surgery to correct strabismus and improve appearance and function may, in part, explain the increase in adult strabismus surgery observed since 2000.

Alternative explanations include an increase in the number of people with strabismus; a reduction in the minimum angle of deviation that would be considered for surgery; an upward shift in the age distribution of the adult 
population, leading to a greater risk of decompensated strabismus and strabismus resulting from vascular complications; ${ }^{15,16}$ and the availability of botulinum toxin. The use of botulinum toxin, as well as being an alternative to surgery, enables assessment of patient satisfaction, the risk of diplopia and quality of binocular vision that can be achieved before committing to having surgery. This is likely to drive the uptake of strabismus surgery in adults. Because we don't know which patients diagnosed with strabismus go on to have strabismus surgery, and if so when, it is not possible for us to say for certain whether or not the increase in adult strabismus surgery is due to more surgery being performed on patients diagnosed with paralytic strabismus or other types of strabismus. Use of the HSCIC bespoke linking service would enable this to be determined in future work. Individuals developing strabismus in adulthood are more likely to suffer from symptoms such as diplopia ${ }^{19}$ and will be more aware of potential benefits arising from correction of the deviation (e.g. improved binocularity and better cosmesis). Nevertheless, regardless of the age of onset of strabismus, there are significant functional and psychosocial benefits to adult strabismus surgery. ${ }^{4-6,19,23}$

We note that trends in treatments in England determined from HES should be interpreted with some caution. Neither FCE's or admissions data from HES reflect the true number of patients, since a given patient may be admitted and treated more than once in a single year. Changes in coding procedures or errors in coding can contribute to changes in time series results. For example, some hospitals use a 01/01/1900 or 01/01/1901 date of birth as default. An increase in this activity may have increased the number of adult strabismus operations recorded over time. Improvements in coverage in HES may also lead to increases in a given activity. Improved recording was incentivised by the Payment by Results scheme starting in 2003 and may have lead to some observed increases in treatments. This system compensated hospitals based on the number and complexity of patient treatments. It is not possible to determine the extent to which any of these factors may have contributed to changes in the frequency of strabismus surgery in adults during the time period analysed. However the trends observed appear gradual, making it unlikely that they are due to changes in coding procedures, new coding schemes, organisational changes, or administrative factors, which might be expected to cause more abrupt changes.

Our findings confirm and extend the previous work investigating trends in paediatric strabismus surgery and provide new and important insights into strabismus surgery rates for adults. The results have implications for the planning of resources and services to ensure adequate delivery of care in the NHS. The reduction in childhood strabismus surgery may suggest a declining need for resources, but consideration of the data for adult strabismus surgery shows that maintenance of service provision for strabismus surgery is important. If the rate of decrease in childhood strabismus surgery continues to slow while the frequency of adult strabismus surgery continues to rise, it will be important to ensure that there are a sufficient number of ophthalmologists, with the necessary training and experience, to deal with the increase in volume. 


\section{References}

1. Roberts J, Rowland M, Statistics NCfH. Refraction status and motility defects of persons 4-74 years, United States, 1971-1972: US Department of Health, Education, and Welfare, Public Health Service, National Center for Health Statistics; 1978.

2. Mills MD, Coats DK, Donahue SP, Wheeler DT. Strabismus surgery for adults: a report by the American Academy of Ophthalmology. Ophthalmology. 2004;111(6):1255-62.

3. Durnian JM, Noonan CP, Marsh IB. The psychosocial effects of adult strabismus: a review. Br J Ophthalmol. 2011;95(4):450-3.

4. Beauchamp GR, Felius J, Stager Sr DR, Beauchamp CL. The utility of strabismus in adults. Trans Am Ophthalmol Soc. 2005;103:164.

5. Pineles SL, Demer JL, Isenberg SJ, Birch EE, Velez FG. Improvement in binocular summation after strabismus surgery. JAMA Ophthalmol. 2015;133(3):326-32.

6. Morris RJ, Scott WE, Dickey CF. Fusion after surgical alignment of longstanding strabismus in adults. Ophthalmology. 1993;100(1):135-8.

7. Gill M, Drummond G. Indications and outcomes of strabismus repair in visually mature patients. Canadian journal of ophthalmology Journal canadien d'ophtalmologie. 1997;32(7):43640.

8. Carney C, Lysons D, Tapley J. Is the incidence of constant esotropia in childhood reducing? Eye (London, England). 1994;9:40-1.

9. Heng SJ, MacEwen CJ. Decrease in the rate of esotropia surgery in the United Kingdom from 2000 to 2010. Br J Ophthalmol. 2013;97(5):598-600.

10. MacEwen CJ, Chakrabarti HS. Why is squint surgery in children in decline? Br J Ophthalmol. 2004;88(4):509-11.

11. Arora A, Williams B, Arora AK, McNamara R, Yates J, Fielder A. Decreasing strabismus surgery. Br J Ophthalmol. 2005;89(4):409-12.

12. Chou MR, Malik AN, Suleman M, Gray M, Yeates D, Goldacre MJ. Time trends over five decades, and recent geographical variation, in rates of childhood squint surgery in England. Br J Ophthalmol. 2013;97(6):746-51.

13. Maino AP, Dawson EM, Lee JP. Strabismus Surgery in the Over 60s-An Update.

Strabismus. 2011;19(1):1-4.

14. Dawson E, Bentley C, Lee J. Squint surgery in the over sixties. Strabismus. 2001;9(4):217-

20.

15. Repka MX, Yu F, Coleman A. Strabismus among aged fee-for-service Medicare

beneficiaries. J AAPOS. 2012;16(6):495-500.

16. Repka MX. Strabismus surgery among aged Medicare beneficiaries. Journal of American Association for Pediatric Ophthalmology and Strabismus. 1997;1(4):231-4.

17. Health and Social Care Information Centre. Hospital Episode Statistics, Admitted Patient Care - England, 2005-2015. Copyright (C) 2015, Re-used with the permission of the Health and Social Care Information Centre. All rights reserved.

18. Office for National Statistics, Population Estimates for UK, England, Wales, Scotland and Northern Ireland, Adapted from data from the Office for National Statistics licensed under the Open Government Licence v.3.0.

19. Beauchamp GR, Black BC, Coats DK, Enzenauer RW, Hutchinson AK, Saunders RA, et al. The management of strabismus in adults-I. clinical characteristics and treatment. Journal of American Association for Pediatric Ophthalmology and Strabismus. 2003;7(4):233-40.

20. Dabes EM, Weakley DR, Birch E. Trends in surgical correction of strabismus: a 20-year experience, 1990-2009. J AAPOS. 2011;15(3):219-23.

21. Coats DK, Stager DR, Beauchamp GR, Mazow ML, Paysse EA, Felius J. Reasons for delay of surgical intervention in adult strabismus. Arch Ophthalmol. 2005;123(4):497-9.

22. Kushner BJ. Intractable diplopia after strabismus surgery in adults. Arch Ophthalmol. 2002;120(11):1498-504.

23. Kushner BJ, Morton GV. Postoperative binocularity in adults with longstanding strabismus. Ophthalmology. 1992;99(3):316-9.

24. Menon V, Saha J, Tandon R, Mehta M, Khokhar S. Study of the psychosocial aspects of strabismus. J Pediatr Ophthalmol Strabismus. 2002;39(4):203-8.

25. McBain HB, MacKenzie KA, Hancox J, Ezra DG, Adams GG, Newman SP. What do patients with strabismus expect post surgery? The development and validation of a questionnaire. Br J Ophthalmol. 2015:bjophthalmol-2015-307027.

26. Nassab R, Harris P. Cosmetic surgery growth and correlations with financial indices: a comparative study of the United Kingdom and United States from 2002-2011. Aesthetic Surgery Journal. 2013;33(4):604-8. 
